\title{
In-plane anisotropic optical and mechanical properties of two-dimensional $\mathrm{MoO}_{3}$
}

\author{
Sergio Puebla (iD) ${ }^{1}$, Roberto D'Agosta (iD ${ }^{2,3}$, Gabriel Sanchez-Santolino ${ }^{4}{ }^{4}$, Riccardo Frisenda ${ }^{1}$, Carmen Munuera (iD ${ }^{1}$ and \\ Andres Castellanos-Gomez (iD)
}

\begin{abstract}
Molybdenum trioxide $\left(\mathrm{MoO}_{3}\right)$ in-plane anisotropy has increasingly attracted the attention of the scientific community in the last few years. Many of the observed in-plane anisotropic properties stem from the anisotropic refractive index and elastic constants of the material but a comprehensive analysis of these fundamental properties is still lacking. Here we employ Raman and microreflectance measurements, using polarized light, to determine the angular dependence of the refractive index of thin $\mathrm{MoO}_{3}$ flakes and we study the directional dependence of the $\mathrm{MoO}_{3}$ Young's modulus using the buckling metrology method. We found that $\mathrm{MoO}_{3}$ displays one of the largest in-plane anisotropic mechanical properties reported for 2D materials so far.
\end{abstract}

npj 2D Materials and Applications (2021)5:37; https://doi.org/10.1038/s41699-021-00220-5

\section{INTRODUCTION}

Recently, two-dimensional (2D) materials with an in-plane anisotropy, such as several transition metal chalcogenides, group-VA, black phosphorus and compounds made of two group-VA elements (so called V-V binary materials), have been extensively studied ${ }^{1-21}$. Some of them have demonstrated a great potential in optoelectronics and flexible electronics applications ${ }^{22-25}$, allowing the fabrication of devices with new functionalities (e.g., polarization sensitive photodetectors ${ }^{22,26}$ ) and the observation of quasi one-dimensional physics phenomena ${ }^{27}$. Among the available anisotropic 2D materials, molybdenum trioxide is a wide band gap semiconductor $(>2.7 \mathrm{eV})^{28}$, which makes it quasi-transparent in the visible spectrum while being still electrically conductive. This nanomaterial has been proven useful for gas sensing ${ }^{29}$, resistive memory technology ${ }^{30}$, optoelectronic $^{31-40}$, electrochromic ${ }^{41}$ and flexible ${ }^{42,43}$ applications. The difference between the in-plane $(a-c)$ lattice parameters makes $\mathrm{a}-\mathrm{MoO}_{3}$ a perfect candidate to investigate optical, mechanical, and electrical in-plane anisotropic properties. Indeed, calculations show that in-plane carrier mobility exhibit strong anisotropic behavior ${ }^{44}$ and highly anisotropic propagation of phonon polaritons have been recently observed in $\mathrm{a}-\mathrm{MoO}_{3}{ }^{45-48}$. In comparison with metallic plasmon polaritons, phonon polaritons can achieve reduced optical losses, improved light confinements and higher quality factors ${ }^{49-52}$. These interesting optical properties are mainly caused by the anisotropy of the fundamental properties of molybdenum trioxide, which until now have been scarcely investigated.

Here, using a combination of experiments and density functional theory calculations, we have studied the directiondependent refractive index (birefringence) and Young's Modulus of $\mathrm{a}-\mathrm{MoO}_{3}$ exfoliated flakes, two fundamental quantities that govern the anisotropy observed in Raman and phonon polariton experiments. We studied thin flakes of $\mathrm{a}-\mathrm{MoO}_{3}$ by aberrationcorrected scanning transmission electron microscopy and energydispersive $\mathrm{x}$-ray spectroscopy. We then deposited the flakes on
$\mathrm{SiO}_{2} / \mathrm{Si}$ substrates and identified the crystallographic orientation of samples using angle-resolved polarized Raman spectroscopy technique ${ }^{53}$. Then, we determined the in-plane anisotropy of the $\mathrm{MoO}_{3}$ refractive index using angle-resolved polarized microreflectance spectroscopy, finding a remarkably large birefringence. The anisotropic mechanical properties of the $\mathrm{MoO}_{3}$ flakes was experimentally investigated with the buckling metrology method $^{54,55}$, finding one of the largest Young's modulus anisotropy reported so far for $2 \mathrm{D}$ materials.

\section{RESULTS}

\section{Crystal structure study}

We have grown $\mathrm{MoO}_{3}$ flakes in atmospheric conditions using a modified version of the hot plate-based physical vapor transport method described in ref. ${ }^{56}$. Briefly, a molybdenum foil was oxidized on a hotplate at $540{ }^{\circ} \mathrm{C}$, then a silicon wafer was placed on top. At this temperature, the molybdenum oxide sublimes and re-crystallizes on the surface of the Si wafer, which is at a slightly lower temperature, forming $\mathrm{MoO}_{3}$ flakes. Then, a Gel-Film (Gel-Pak WF $\times 46.0$ mil) stamp is used to pick-up and exfoliate the $\mathrm{MoO}_{3}$ flakes. These flakes can be then transferred onto a target substrate (e.g., a holey $\mathrm{Si}_{3} \mathrm{~N}_{4}$ TEM grid or a $297 \mathrm{~nm} \mathrm{SiO} / 2$ Si substrate) using deterministic transfer set up (see Materials and Methods section for more information).

Figure 1 a shows the layered crystal structure of $\mathrm{a}-\mathrm{MoO}_{3}{ }^{57-59}$, belonging to the Pbnm space group. It consists of a double-layer stacking of linked distorted $\mathrm{MoO}_{6}$ octahedra in the $b$ direction, along which the adjacent layers are linked by weak van der Waals forces, while in-plane atoms are strongly bonded. This configuration leads to lattice parameters: $a=3.96 \AA, b=13.86 \AA$ and $c=$ $3.70 \AA$ (JCPDS file: $05-0508)^{44,53,56,59,60}$. We characterized the structure and composition of the grown $\mathrm{MoO}_{3}$ flakes using scanning transmission electron microscopy (STEM) along with energy dispersive x-ray spectroscopy (EDS) and scanning electron microscopy (see Supplementary Fig. 4 in the Supporting

\footnotetext{
${ }^{1}$ Materials Science Factory. Instituto de Ciencia de Materiales de Madrid (ICMM-CSIC), Madrid, Spain. ${ }^{2}$ Nano-Bio Spectroscopy Group and European Theoretical Spectroscopy Facility (ETSF), Departamento Polímeros y Materiales Avanzados: Física, Química y Tecnología, Universidad del País Vasco UPV/EHU, San Sebastián, Spain. ${ }^{3}$ IKERBASQUE, Basque Foundation for Science, Bilbao, Spain. ${ }^{4}$ GFMC, Departamento de Física de Materiales \& Instituto Pluridisciplinar, Universidad Complutense de Madrid, Madrid, Spain.

$凶_{\text {email: andres.castellanos@csic.es }}$
} 
(a)
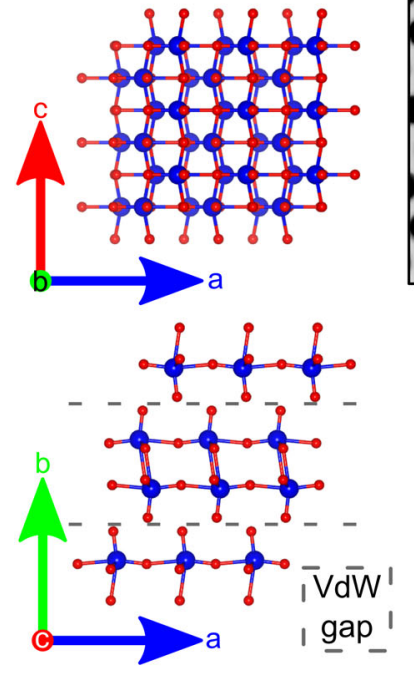

(b)

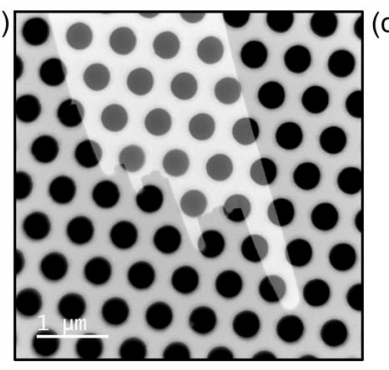

(e)
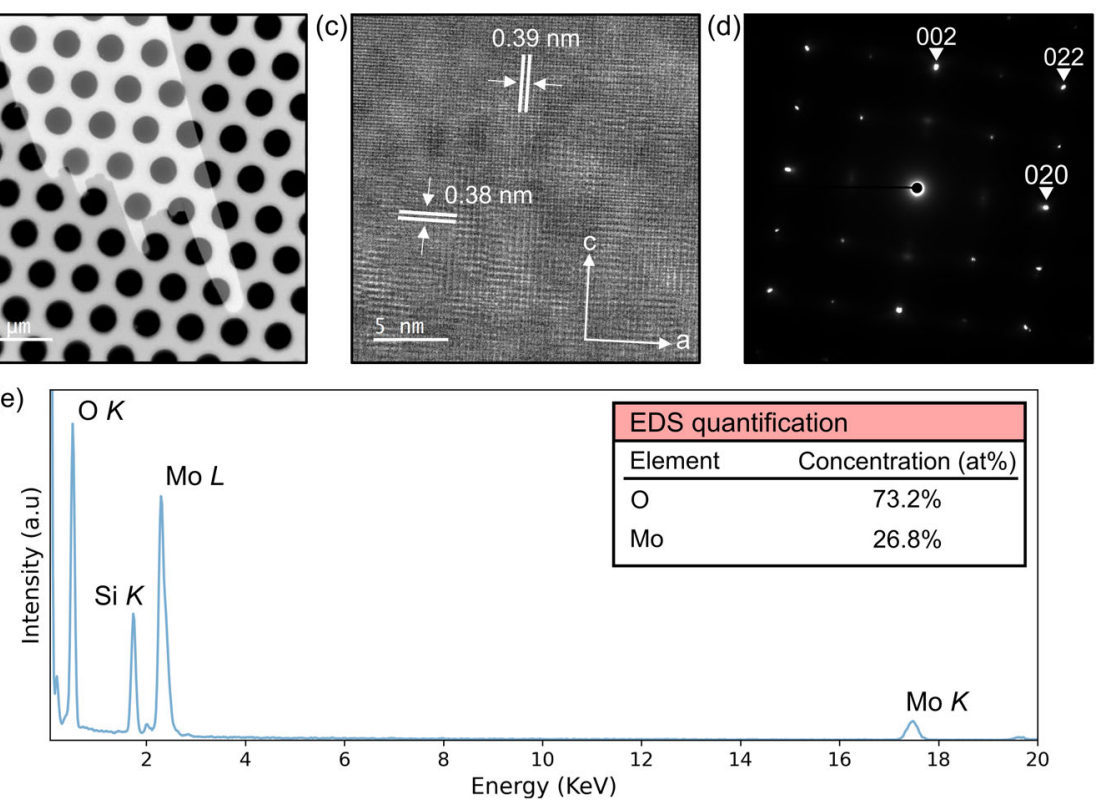

Fig. 1 Anisotropic structure of $\mathrm{MoO}_{3}$ flakes. a Crystal structure of $\mathrm{MoO}_{3}$ in $\mathrm{Pbnm}$ notation, spheres in blue (red) represent $\mathrm{Mo}(\mathrm{O})$ atoms, the two different views belong to ca (top), and ba plane projection (bottom). b Low magnification HAADF image of a mechanically exfoliated $\mathrm{MoO}_{3}$ flake transferred over a porous SiN membrane, scale bar is of $1 \mu \mathrm{m}$. c Atomic resolution HAADF image depicting the anisotropy between the in-plane (a-c) lattice parameters, scale bar is of $5 \mathrm{~nm}$. d SAED pattern characteristic of the orthorhombic $\alpha-\mathrm{MoO}_{3}$. e EDS spectrum taken at the flake shown in $\mathbf{b}$.

Information). A thin flake was transferred into a porous $\mathrm{Si}_{3} \mathrm{~N}_{4}$ membrane, as shown in Fig. 1b. Atomic resolution high angle annular dark field (HAADF) imaging shows an orthorhombic a- $\mathrm{MoO}_{3}$ structure with a difference in the $a$-c lattice parameters, as depicted also in a selected area diffraction pattern (SAED) acquired at the same flake (Fig. 1C, d) $44,53,56,59,60$. The chemical composition of the flake was determined by EDS, Fig. 1e shows the spectrum of $\mathrm{MoO}_{3}$ used for quantification, where we find a small oxygen deficiency.

\section{Angle-resolved polarized Raman spectroscopy analysis}

We have used angle-resolved polarized Raman spectroscopy technique to identify the different crystal directions in our $\mathrm{MoO}_{3}$ flakes. Using a linearly polarized laser in a Raman system (see Materials and Methods section) and setting the analyzer and polarizer in a parallel configuration, while we rotate the sample, we obtain the spectra shown in Fig. 2a. The system set up is depicted in Supplementary Fig. 1 in the Supplementary Information and it is explained in detail by Liu et al. ${ }^{61}$. Typical $\mathrm{MoO}_{3}$ Raman modes are $\mathrm{A}_{\mathrm{g}}$ and $\mathrm{B}_{\mathrm{g}}{ }^{62}$ and no correlation has been found between the Raman modes and the $\mathrm{MoO}_{3}$ thickness ${ }^{63}$. We highlight the peaks centered at $282 \mathrm{~cm}^{-1}$, assigned to $B_{2 g}$ mode, and at 156 and $818 \mathrm{~cm}^{-1}$, assigned to $A_{g}{ }^{c}$ and $A_{g}{ }^{a}$ mode, respectively. The $A_{g}{ }^{c}$ peak corresponds to the translation vibration of the rigid $\mathrm{MoO}_{6}$ octahedra chains along the c-axis, and the $\mathrm{A}_{\mathrm{g}}{ }^{\mathrm{a}}$ mode is the asymmetric stretching vibration of O-Mo-O atoms along the a-axis ${ }^{53,62}$.

We have carried out angle-resolved polarized Raman measurements in $\mathrm{a} \mathrm{MoO}_{3}$ flake of $28 \mathrm{~nm}$ of thickness from $0^{\circ}$ to $360^{\circ}$, with a step of $4^{\circ}$. The thickness has been determined by combination of atomic force microscopy with recently developed optical microscopy based techniques ${ }^{64}$. In Fig. 2 a three of these measurements at different angles $\left(0^{\circ}, 45^{\circ}\right.$ and $\left.90^{\circ}\right)$ are displayed. Notice the difference in intensity of each mode, revealing how the relative orientation between the incident laser polarization angle and the direction of the crystalline axes plays an important role in the Raman process. In fact, previous works have shown how the $A_{g}$ and $B_{2 g}$ mode intensities can be fitted to ${ }^{53,62}$ :

$$
\begin{aligned}
& I\left(A_{g}\right) \propto\left(A \cos ^{2} \beta+C \sin ^{2} \beta\right)^{2} \\
& I\left(B_{2 g}\right) \propto E^{2} \sin ^{2} 2 \beta
\end{aligned}
$$

where $\beta$ is the relative angle between the a-axis crystal direction and the linear polarization direction of the laser. Figure $2 b$ shows the normalized intensity angle-resolved polarized Raman measurements of each mode (light red), with the resulting fit to Eqs. (1) and (2) (in dark red), showing an excellent agreement. Note, the $A_{g}{ }^{c}$ and $A_{g}{ }^{a}$ modes are useful to extract the crystal structure of the sample due to their 180-degree periodicity. The inset in Fig. 2a shows a microscopy image of the $\mathrm{MoO}_{3}$ flake on $\mathrm{SiO}_{2} / \mathrm{Si}$, studied with the Raman spectroscopy. The a- and c-axes, determined from the Raman measurements are depicted in the figure and coincide with the straight edges produced during the exfoliation of the $\mathrm{MoO}_{3}$ flake.

\section{Polarized micro-reflectance analysis}

In order to gain an insight about the in-plane anisotropic optical properties of $\mathrm{MoO}_{3}$ flakes we carried out micro-reflectance measurements employing linearly polarized incident light. Figure 3 shows the optical contrast spectra acquired for different alignment between the crystal axis and the incident linear polarization (see the bottom inset). The optical contrast $C$ is defined as:

$C=\frac{l_{f l}-I_{\text {sub }}}{I_{\text {fl }}+I_{\text {sub }}}$

where $I_{f l}$ and $I_{\text {sub }}$ are the intensity measured on the $\mathrm{MoO}_{3}$ flake and on the bare substrate, respectively. Interestingly, it has been demonstrated how one can use a simple Fresnel law-based model to accurately reproduce the measured optical contrast spectra ${ }^{64}$. Moreover, given the known refractive indexes of air, $\mathrm{SiO}_{2}$ and $\mathrm{Si}$ and the known thickness of the $\mathrm{SiO}_{2}$ film and $\mathrm{MoO}_{3}$ flake one can determine the refractive index of the $\mathrm{MoO}_{3}$ flake for different alignment between the incident linearly polarized light and the crystal directions by using the refractive index as a fitting 

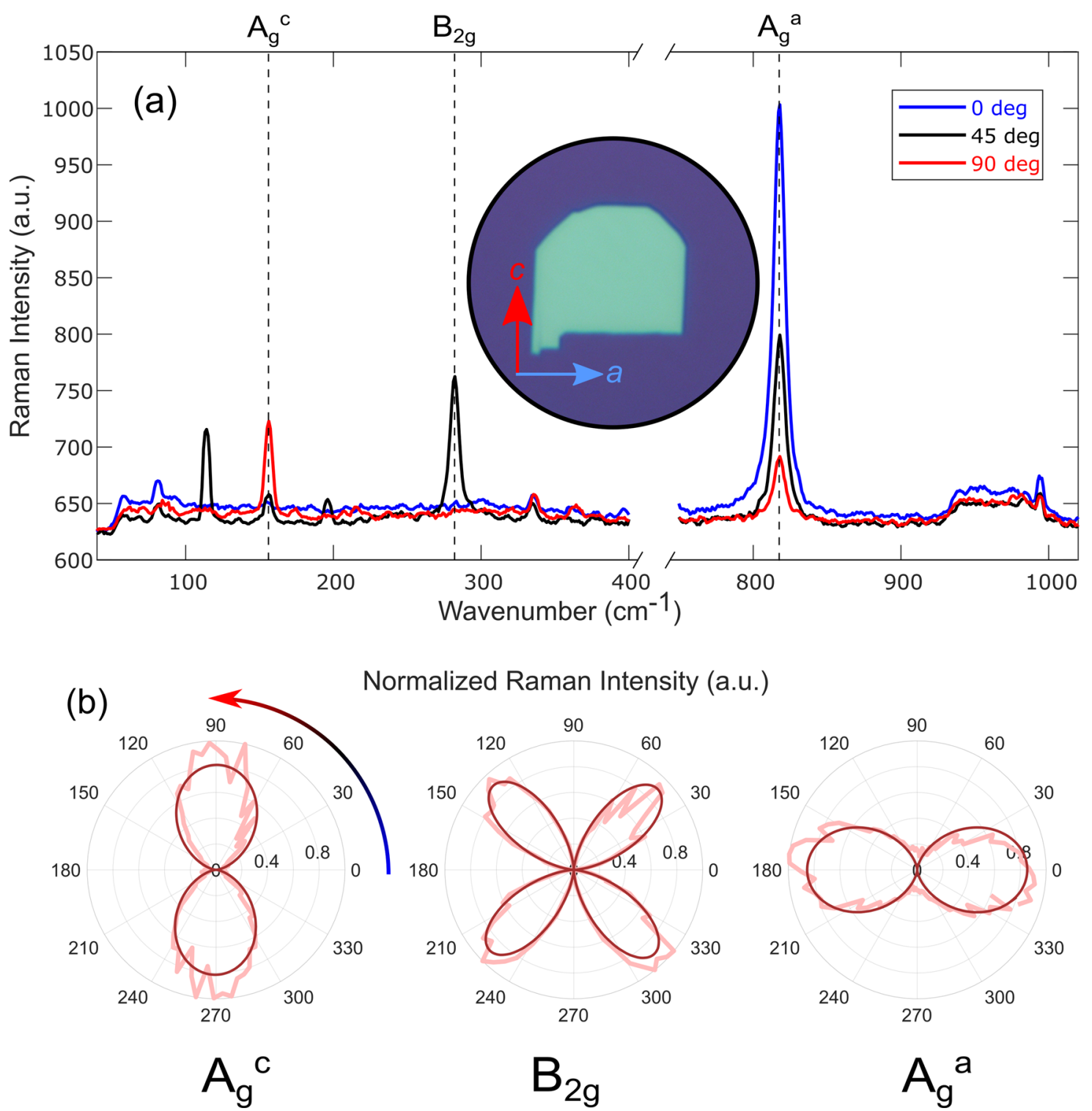

Fig. 2 Determining the crystal orientation of $\mathrm{MoO}_{3}$ through Raman spectroscopy. a Raman spectra at $0^{\circ}$ (blue), $45^{\circ}$ (black) and $90^{\circ}$ (red) angles with respect to the horizontal axis. $A_{g}{ }^{c}, B_{2 g}$ and $A_{g}{ }^{a}$ Raman modes are highlighted with vertical dashed lines. Inset: Microscopy image of the sample placed in the initial position $\left(0^{\circ}\right)$ and the $a$ and $c$ axes, determined from the angle-resolved Raman measurements, are shown. b Polar plots, in light red; and fittings, in dark red, of angle-resolved normalized Raman intensities of the $A_{g}{ }^{c}$ (left), $B_{2 g}(m i d d l e)$ and $A_{g}{ }^{a}$ (right) modes.

parameter to achieve the best fit of the Fresnel law-based model to the experimental data. The top inset in Fig. 3 shows the resulting in-plane angular dependence of the refractive index displaying a marked anisotropy (birefringence). While along the aaxis the refractive index of $\mathrm{MoO}_{3}$ is $n_{\mathrm{a}}=2.21+0 \mathrm{i}$, along the c-axis the refractive index increases up to $n_{c}=2.30+0$ i. The difference $\Delta n$ between $n_{\mathrm{c}}$ and $n_{\mathrm{a}}$, is $\sim 0.1$. This value is comparable to that of well-known strongly birefringent materials like calcite $(-0.17)$ and barium borate $(-0.12)^{65,66}$. If we compare it with other anisotropic 2D materials, the birefringence of $\mathrm{MoO}_{3}$ is larger than that of $\mathrm{ReS}_{2}$ and $\operatorname{ReSe}_{2}(\sim 0.04)^{6}$ but substantially lower than the values reported for black phosphorus $(0.25)^{6}$ or $\mathrm{TiS}_{3}(0.3)^{67}$. However, note that, unlike $\mathrm{MoO}_{3}$, all these anisotropic $2 \mathrm{D}$ materials are rather opaque in the visible range of the electromagnetic spectrum, limiting their application in polarization optics applications. Therefore, the birefringence value of $\mathrm{MoO}_{3}$, although more modest in comparison with $\mathrm{TiS}_{3}$ or black phosphorus, can have a stronger impact in future ultrathin polarization optics applications.
These experimental results for $\mathrm{MoO}_{3}$ are confirmed by theoretical ab-initio calculations through the solution of the Bethe-Salpeter's equation, in which we have obtained refractive index values of $n_{a}=2.40$ and $n_{\mathrm{c}}=2.60$ at small frequency, with a difference $\Delta \mathrm{n} \sim$ 0.2 . We find these theoretical values close to the ones obtained experimentally. We attribute the lower birefringence experimental value to the presence of defects in the $\mathrm{MoO}_{3}$ flakes, not considered in the calculations, that can effectively reduce the anisotropy of the lattice.

\section{Mechanical anisotropy study}

In the following we focus on the characterization of the anisotropy of the Young's modulus, one of the fundamental magnitudes that govern the mechanical properties of materials, of $\mathrm{MoO}_{3}$ flakes. We use buckling induced metrology method, which has been proved to be an easy, but reliable way to study the Young's modulus of thin films $s^{54,55}$ and 2D materials ${ }^{68-72}$. The method relies on studying the buckling instability that arises when a thin film is 


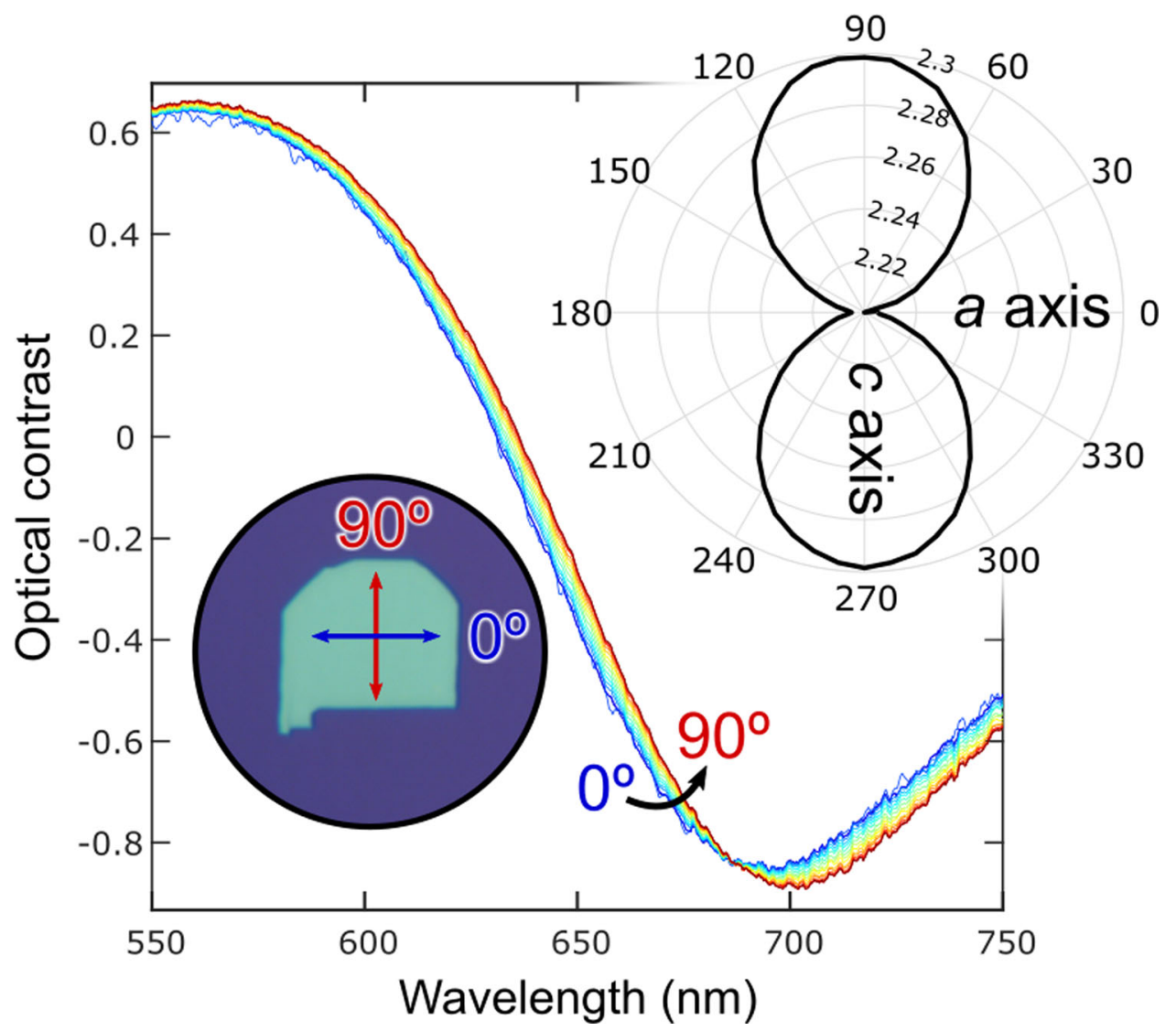

Fig. 3 Birefringence of $\mathrm{MoO}_{3}$ flakes. Optical contrast vs. wavelength spectra, measured for the $28 \mathrm{~nm}$ thick $\mathrm{MoO}_{3}$ flake shown in the bottom inset, as function of the angle formed between the incident linearly polarized light and the crystal a-axis. Top inset: Polar plot of the change in refractive index as a function of the relative orientation between the incident linearly polarized light and the a-axis of the crystal.

deposited onto an adhesive compliant substrate, and it is subjected to in-plane uniaxial compression ${ }^{73}$. Because of this compression, there is a trade-off in the energy related to the adhesion forces between film and substrate and the bending rigidity of the film. This trade-off leads to a rippling pattern of the thin film, which is characteristic of the film and substrate properties (in the Supplementary Information it can be found an animated GIF, Supplementary GIF, of a $\mathrm{a}-\mathrm{MoO}_{3}$ flake compressed in this way). To perform the buckling metrology measurements, the $\mathrm{MoO}_{3}$ flakes were transferred onto a flat (unstrained) Gel-Film substrate that is mounted on a rotation stage under the inspection of an optical microscope. Then the flakes were subjected to compressive strain along different crystal directions by pinching the surface of the Gel-Film with two glass slides and pictures of the obtained ripple patterns are acquired with a digital camera attached to the microscope. The $\mathrm{MoO}_{3}$ flakes are then transferred to a $\mathrm{SiO}_{2} / \mathrm{Si}$ substrate to determine their crystal orientation, through Raman spectroscopy and micro-reflectance, and thickness through AFM (see Supplementary Figs. 2 and 3 of the Supporting Information).

The wavelength, $\lambda$, of the rippling pattern can be used to determine the Young's modulus:

$E_{f}=3\left(\frac{\lambda}{2 \pi h}\right)^{3} \frac{1-v_{f a} v_{f c}}{1-v_{s}^{2}} E_{s}$

being $h$ the flake thickness, $v_{s}, v_{f}, E_{s}$ and $E_{f}$ the Poisson's ratio and Young's Modulus of substrate and flake, respectively. The Poisson's ratio of $\mathrm{a}-\mathrm{MoO}_{3}$ must be taken along the two axes. Using density functional theory (more details available in the Supplementary Information) we calculate Poisson's ratios of
$\mathrm{a}-\mathrm{MoO}_{3}$ correspond to $\mathrm{v}_{\mathrm{fa}}=0.147$ and $\mathrm{v}_{\mathrm{fc}}=0.06$. In our experiment we used a Gel-Film substrate as compliant substrate, with $v_{\text {sub }}=0.5^{74}$ and its Young's Modulus is $E_{\text {sub }}=492 \pm 11 \mathrm{kPa}^{68}$.

Figure $4 \mathrm{a}$ shows optical images of a $24 \mathrm{~nm}$ thick $\mathrm{MoO}_{3}$ flake (thickness and orientation obtained with optical microscopy based technique, shown in Supplementary Fig. 2) when it is subjected to compression along the a- and c-axes. Upon compression along different orientations the $\mathrm{MoO}_{3}$ develops a rippled pattern whose periodicity depends on the direction. In Fig. 4b, a statistical study is shown with 14 different samples with thicknesses ranging from 18 to $62 \mathrm{~nm}$ (white circles), from which we obtain the mean Young's Modulus values and their standard deviation along the aand c-axis. We use histograms to show the flake-to-flake variability of these results and we fit them with a normalized Gaussian distribution function. Moreover, we plot the corresponding twodimensional normalized Gaussian distribution function in a 2D gray colormap, in which the density of datapoints is associated with the colorbar, set as inset. The obtained Young's modulus values along the a- and c-axes directions are $E_{\mathrm{a} \text {-axis }}=44 \pm 8 \mathrm{GPa}$ and $E_{\text {c-axis }}=86 \pm 15 \mathrm{GPa}$, respectively. Interestingly the anisotropy ratio $\left(E_{\mathrm{c} \text {-axis }} / E_{\mathrm{a} \text {-axis }}\right)$ is $\sim 2$ among the largest value reported in the literature for anisotropic $2 \mathrm{D}$ materials: black phosphorus $\sim 2.7^{17}$, for orpiment $\left(\mathrm{As}_{2} \mathrm{~S}_{3}\right)$ is $\sim 1.7^{75}$. Our DFT calculations predict even higher Young's modulus values (91.9 GPa and $216.9 \mathrm{GPa}$ for the aand c-axis respectively) in relatively good agreement with the values reported for bulk-like $\mathrm{MoO}_{3}(\sim 200 \mathrm{~nm}$ thick) crystals through Brillouin scattering. Note that the presence of defects in the synthesized $\mathrm{MoO}_{3}$ samples, like the oxygen vacancies found in the STEM-EDS analysis, could explain the lower Young's modulus values obtained in our experiments. 

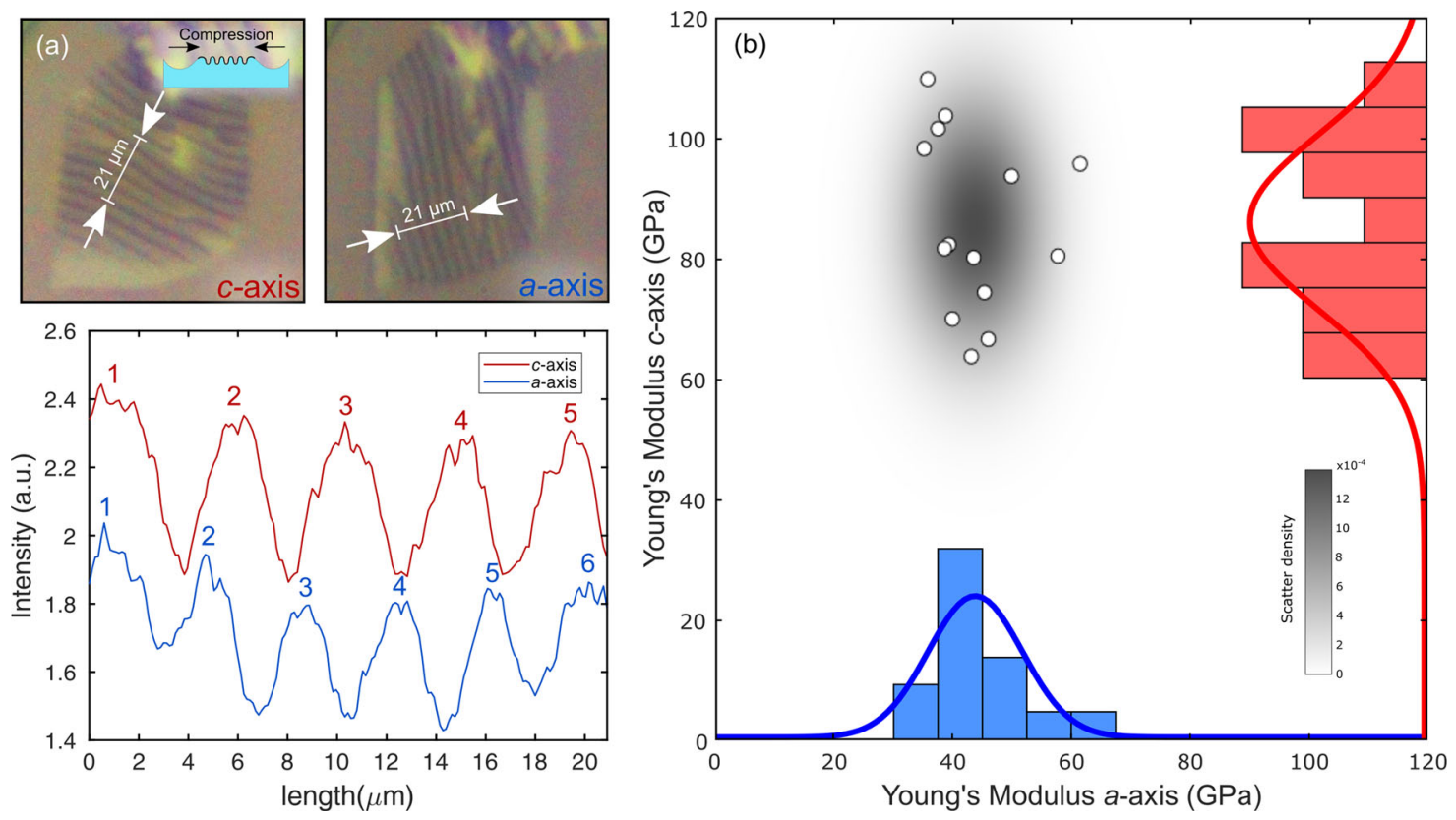

Fig. 4 Anisotropic mechanical properties of $\mathrm{MoO}_{3}$ flakes. a Optical images of a $\mathrm{MoO}_{3}$ flake on Gel-Film substrate applying compression along a and c axes (top images), inset: process of ripples formation; determination of the wavelength of periodic ripples (bottom figure). b Scatter density plot of Young's modulus values along the two directions obtained from 14 different samples, white circles with black edges, and fitted with a multivariate Gaussian normal distribution function, in gray. Inset: Histogram plots of Young's modulus along the a-axis (blue) and the c-axis (red), fitted with a Gaussian distribution.

\section{DISCUSSION}

In summary, we combined scanning transmission electron microscopy, Raman spectroscopy, micro-reflectance and buckling metrology to probe the anisotropic optical and mechanical properties of exfoliated $\mathrm{MoO}_{3}$ flakes. We found that the flakes show a strong birefringence, i.e., their refractive index depends on the alignment between the polarization of the incident light and the crystalline axis. The difference between the refractive index for light polarized along the a- and $c$-axes reaches $\sim 0.1$. This large value, together with the fact that $\mathrm{MoO}_{3}$ is transparent in the visible range, makes this material a good candidate for future polarization optics applications. Regarding the mechanical properties, we found that the experimental Young's modulus along the a- and c-axes directions are $E_{\mathrm{a} \text {-axis }}=44 \pm 8 \mathrm{GPa}$ and $E_{\mathrm{c} \text {-axis }}=86 \pm 15 \mathrm{GPa}$, respectively, yielding an anisotropy ratio of $\sim 2$, only surpassed by black phosphorus. Our results agree with ab-initio calculations of optical and elastic properties of $\mathrm{MoO}_{3}$. The anisotropy in the refractive index and the Young's modulus have strong impact in many optical and mechanical properties and thus, we believe that our work can be used as the foundation of further works studying the intriguing anisotropic properties of $\mathrm{MoO}_{3}$.

\section{METHODS}

\section{Growth and deposition}

We have based our present grown procedure on a modification of the hot plate growth method developed by Molina-Mendoza et al. ${ }^{56}$.

Once the growth of the crystals is finished, the $\mathrm{MoO}_{3}$ flakes are firstly exfoliated onto a polydimethylsiloxane (PDMS) (Gel-Film WF x4 6.0 mil, by $\mathrm{Gelpak}^{\circledR}$ ) and then transferred onto a $297 \mathrm{~nm} \mathrm{SiO}_{2} / \mathrm{Si}$ substrate using a deterministic transfer method ${ }^{76,77}$.

\section{Scanning transmission electron microscopy}

For the scanning transmission electron microscope characterization, we used an aberration-corrected JEOL JEM-ARM 200cF electron microscope operated at $80 \mathrm{kV}$, equipped with a cold field emission gun and an Oxford Instruments EDS spectrometer.

\section{Optical microscopy and spectroscopy}

Optical microscopy images were acquired using a Motic BA310 MET-T microscope equipped with a $50 \times 0.55$ NA objective and an AMScope MU1803 CMOS Camera. Reflection spectra were collected from a spot of $\sim 1.5-2 \mu \mathrm{m}$ diameter with a Thorlabs CCS200/M fiber-coupled spectrometer (Thorlabs Inc., Newton, New Jersey, United States). More details about the micro-reflectance setup can be found in Reference ${ }^{78}$.

\section{Raman spectroscopy}

Raman characterization of $\mathrm{MoO}_{3}$ flakes on $297 \mathrm{~nm} \mathrm{SiO} / 2 / \mathrm{Si}$ substrates were carried out with a confocal Raman microscopy system (MonoVista CRS+ from Spectroscopy \& Imaging $\mathrm{GmbH}$ ) using a $532 \mathrm{~nm}$ excitation laser with an incident power of $1.234 \mathrm{~mW}$ and a $100 \times$ objective with the integration time of $20 \mathrm{~s}$.

\section{Numerical methods}

We have analyzed the optical and elastic properties of $\mathrm{MoO}_{3}$ using the YAMBO and Quantum Espresso suite of programs ${ }^{79-81}$. For the electronic structure calculation we have used the generalized gradient approximation in the PBE parametrization for the exchange-correlation energy with a plane wave cut-off of $60 \mathrm{Ry}^{82}$. The electronic structure calculations are performed on a Monkhorst-Pack grid of $8 \times 8 \times 8$ points. We have chosen norm-conserving pseudo potentials in the SG15 database ${ }^{83}$.

For the elastic properties, we have used the thermo pw code from the Quantum Espresso suite ${ }^{84}$. The elastic properties agree when we used either norm-converting or ultra-soft pseudo potentials.

\section{DATA AVAILABILITY}

Data presented in this study are available on request from the authors.

Received: 20 January 2021; Accepted: 16 March 2021; Published online: 12 April 2021

\section{REFERENCES}

1. Wei, Q. \& Peng, X. Superior mechanical flexibility of phosphorene and few-layer black phosphorus. Appl. Phys. Lett. 104, 251915 (2014). 
2. Zheng, Q. et al. In-plane anisotropic raman response and electrical conductivity with robust electron-photon and electron-phonon interactions of air stable $\mathrm{MoO}_{2}$ nanosheets. J. Phys. Chem. Lett. 10, 2182-2190 (2019).

3. Huang, S. et al. In-plane optical anisotropy of layered gallium telluride. ACS Nano 10, 8964-8972 (2016).

4. Zhou, X. et al. Ultrathin $2 \mathrm{D}$ GeSe2 rhombic flakes with high anisotropy realized by Van der Waals epitaxy. Adv. Funct. Mater. 27, 1-9 (2017).

5. Kong, W. et al. Angle resolved vibrational properties of anisotropic transition metal trichalcogenide nanosheets. Nanoscale 9, 4175-4182 (2017).

6. Yang, $\mathrm{H}$. et al. Optical waveplates based on birefringence of anisotropic twodimensional layered materials. ACS Photonics 4, 3023-3030 (2017).

7. Chenet, D. A. et al. In-plane anisotropy in mono- and few-layer ReS2 probed by raman spectroscopy and scanning transmission electron microscopy. Nano Lett. 15, 5667-5672 (2015)

8. Wolverson, D., Crampin, S., Kazemi, A. S., llie, A. \& Bending, S. J. Raman spectra of monolayer, few-layer, and bulk ReSe2: an anisotropic layered semiconductor. ACS Nano 8, 11154-11164 (2014).

9. Hafeez, M., Gan, L., Li, H., Ma, Y. \& Zhai, T. Chemical vapor deposition synthesis of ultrathin hexagonal ReSe2 flakes for anisotropic raman property and optoelectronic application. Adv. Mater. 28, 8296-8301 (2016).

10. Xiong, W., Huang, K. \& Yuan, S. The mechanical, electronic and optical properties of two-dimensional transition metal chalcogenides $M X 2$ and $M 2 X 3(M=N i, P d$; $\mathrm{X}=\mathrm{S}, \mathrm{Se}, \mathrm{Te}$ ) with hexagonal and orthorhombic structures. J. Mater. Chem. C. 7, 13518-13525 (2019).

11. Zhang, S. et al. Recent progress in $2 D$ group-VA semiconductors: from theory to experiment. Chem. Soc. Rev. 47, 982-1021 (2018).

12. Zhao, P. et al. Giant anisotropic photogalvanic effect in a flexible AsSb monolayer with ultrahigh carrier mobility. Phys. Chem. Chem. Phys. 19, 27233-27239 (2017).

13. Li, L. et al. Emerging in-plane anisotropic two-dimensional materials. 54-73, https://doi.org/10.1002/inf2.12005 (2019).

14. Guo, S. et al. 2D V-V binary materials: status and challenges. Adv. Mater. 31, 1-19 (2019).

15. Frisenda, R. et al. Symmetry breakdown in franckeite: spontaneous strain, rippling, and interlayer Moiré. Nano Lett. 20, 1141-1147 (2020).

16. Xia, F., Wang, H. \& Jia, Y. Rediscovering black phosphorus as an anisotropic layered material for optoelectronics and electronics. Nat. Commun. 5, 1-6 (2014).

17. Vaquero-Garzon, L., Frisenda, R. \& Castellanos-Gomez, A. Anisotropic buckling of few-layer black phosphorus. Nanoscale 11, 12080-12086 (2019).

18. Island, J. O. et al. Titanium trisulfide (TiS3): a $2 \mathrm{D}$ semiconductor with quasi-1D optical and electronic properties. Sci. Rep. 6, 1-7 (2016)

19. Beams, R. et al. Characterization of few-layer $1 \mathrm{~T}^{\prime} \mathrm{MoTe} 2$ by polarization-resolved second harmonic generation and Raman scattering. ACS Nano 10, 9626-9636 (2016).

20. Qiu, G. et al. Observation of optical and electrical in-plane anisotropy in highmobility few-layer ZrTe 5. Nano Lett. https://doi.org/10.1021/acs. nanolett.6b02629 (2016).

21. Li, L. et al. Strong in-plane anisotropies of optical and electrical response in layered dimetal chalcogenide. ACS Nano 11, 10264-10272 (2017).

22. Yuan, $\mathrm{H}$. et al. Polarization-sensitive broadband photodetector using a black phosphorus vertical p-n junction. Nat. Nanotechnol. 10, 707-713 (2015).

23. Wang, $X$. et al. Short-wave near-infrared linear dichroism of two-dimensional germanium selenide. J. Am. Chem. Soc. 139, 14976-14982 (2017).

24. Gong, C. et al. Electronic and optoelectronic applications based on 2D novel anisotropic transition metal dichalcogenides. Adv. Sci. 4, 1700231 (2017).

25. Zhao, Q. et al. Flexible and anisotropic properties of monolayer $M X 2(M=T c$ and $\mathrm{Re} ; \mathrm{X}=\mathrm{S}, \mathrm{Se}$ ). J Phys. Chem. 2, 23744-23751 (2017).

26. Niu, Y. et al. Polarization-sensitive and broadband photodetection based on a mixed-dimensionality TiS3/Si p-n junction. Adv. Opt. Mater. 6, 1-7 (2018).

27. Xing, B., Yu, Z., Fu, L., Qin, Q. \& Lu, Y. Extraordinarily bound quasi-one-dimensional trions in two-dimensional phosphorene atomic semiconductors. ACS Nano. https://doi.org/10.1021/acsnano.5b06193 (2016).

28. Bouzidi, A. et al. Effect of substrate temperature on the structural and optical properties of $\mathrm{MoO}_{3}$ thin films prepared by spray pyrolysis technique. Mater. Sci. Eng. B Solid-State Mater. Adv. Technol. 97, 5-8 (2003).

29. Rahman, F. et al. Dual selective gas sensing characteristics of $2 \mathrm{D}$ a- $-\mathrm{MoO}_{3-\mathrm{x}}$ via a facile transfer process. ACS Appl. Mater. Interfaces 11, 40189-40195 (2019).

30. Rahman, F. et al. Reversible resistive switching behaviour in CVD grown, large area MoOX. Nanoscale 10, 19711-19719 (2018).

31. Guo, Y. et al. Eighteen functional monolayer metal oxides: wide bandgap semiconductors with superior oxidation resistance and ultrahigh carrier mobility. Nanoscale Horiz. 4, 592-600 (2019).

32. Balendhran, S. et al. Enhanced charge carrier mobility in two-dimensional high dielectric molybdenum oxide. Adv. Mater. 25, 109-114 (2013).

33. Kanai, K. et al. Electronic structure of anode interface with molybdenum oxide buffer layer. Org. Electron. 11, 188-194 (2010).
34. Shrotriya, V., Li, G., Yao, Y., Chu, C. W. \& Yang, Y. Transition metal oxides as the buffer layer for polymer photovoltaic cells. Appl. Phys. Lett. 88, 1-4 (2006)

35. You, H., Dai, Y., Zhang, Z. \& Ma, D. Improved performances of organic lightemitting diodes with metal oxide as anode buffer. J. Appl. Phys. 101, 1-4 (2007).

36. $\mathrm{Du}, \mathrm{J}$. et al. Pressure-assisted fabrication of organic light emitting diodes with $\mathrm{MoO}_{3}$ hole-injection layer. J. Appl. Phys. 115, 233703 (2014).

37. Meyer, J., Shu, A., Kröger, M. \& Kahn, A. Effect of contamination on the electronic structure and hole-injection properties of $\mathrm{MoO}_{3}$ /organic semiconductor interfaces. Appl. Phys. Lett. 96, 8-11 (2010).

38. de Castro, I. A. et al. Molybdenum oxides - from fundamentals to functionality. Adv. Mater. 29, 1-31 (2017).

39. Balendhran, S. et al. Large-area synthesis of two-dimensional $\mathrm{MoO}_{3-\mathrm{x}}$ for enhanced optoelectronic applications. 2D Mater. 6, 035031 (2019).

40. Balendhran, S. et al. Two-dimensional molybdenum trioxide and dichalcogenides. Adv. Funct. Mater. 23, 3952-3970 (2013).

41. Arash, A. et al. Electrically Activated UV - A Filters Based on Electrochromic $\mathrm{MoO}_{3-\mathrm{x}}$. ACS Appl. Mater. Interfaces https://doi.org/10.1021/acsami.9b20916 (2020).

42. Cho, E., Cha, S., Kim, Y. \& Kim, C. Transparent and flexible electrode composed of a graphene multilayer interlayer-doped with $\mathrm{MoO}_{3}$. Org. Electron. 77, 105437 (2020).

43. Han, Y. C., Lim, M. S., Park, J. H. \& Choi, K. C. ITO-free flexible organic light-emitting diode using $\mathrm{ZnS} / \mathrm{Ag} / \mathrm{MoO}_{3}$ anode incorporating a quasi-perfect Ag thin film. Org. Electron. 14, 3437-3443 (2013).

44. Zhang, W. B., Qu, Q. \& Lai, K. High-mobility transport anisotropy in few-layer $\mathrm{MoO}_{3}$ and its origin. ACS Appl. Mater. Interfaces 9, 1702-1709 (2017).

45. Ma, W. et al. In-plane anisotropic and ultra-low-loss polaritons in a natural van der Waals crystal. Nature 562, 557-562 (2018).

46. Zheng, Z. et al. A mid-infrared biaxial hyperbolic van der Waals crystal. Sci. Adv. 1-9 eaav8690 (2019).

47. Chen, M. et al. Configurable phonon polaritons in twisted a- $\mathrm{MoO}_{3}$. Nat. Mater. 19, 1307-1311 (2020).

48. Zheng, Z. et al. Phonon polaritons in twisted double-layers of hyperbolic van der Waals crystals. Nano Lett. 20, 5301-5308 (2020).

49. Zheng, Z. et al. Highly confined and tunable hyperbolic phonon polaritons in Van Der Waals semiconducting transition metal oxides. Adv. Mater. 30, 1705318 (2018).

50. Wang, T., Li, P., Hauer, B., Chigrin, D. N. \& Taubner, T. Optical properties of single infrared resonant circular microcavities for surface phonon polaritons. Nano Lett. 13, 5051-5055 (2013)

51. Summary, R., Basov, D. N., Fogler, M. M. \& García De Abajo, F. J. Polaritons in van der Waals materials. Science 354, aag1992 (2016).

52. Low, T. et al. Polaritons in layered two-dimensional materials. Nat. Mater. 16, 182-194 (2016).

53. Zheng, B., Wang, Z., Chen, Y., Zhang, W. \& Li, X. Centimeter-sized 2D a-MoO single crystal: growth, Raman anisotropy, and optoelectronic properties. $2 D$ Mater. 5, 045011 (2018).

54. Stafford, C. M. et al. A buckling-based metrology for measuring the elastic moduli of polymeric thin films. Nat. Mater. 3, 545-550 (2004).

55. Khang, D. Y., Rogers, J. A. \& Lee, H. H. Mechanical buckling: mechanics, metrology, and stretchable electronics. Adv. Funct. Mater. 19, 1526-1536 (2009).

56. Molina-Mendoza, A. J. et al. Centimeter-scale synthesis of ultrathin layered $\mathrm{MoO}_{3}$ by van der Waals Epitaxy. Chem. Mater. 28, 4042-4051 (2016).

57. Carcia, P. F. \& McCarron, E. M. Synthesis and properties of thin film polymorphs of molybdenum trioxide. Thin Solid Films 155, 53-63 (1987).

58. McCarron, E. M. \& Carcia, P. F. Properties of $\mathrm{MoO}_{3}$ thin film polymorphs. Mat. Res. Soc. Symp. Proc. 77, 319-326 (1987).

59. Kalantar-Zadeh, K. et al. Synthesis of nanometre-thick $\mathrm{MoO}_{3}$ sheets. Nanoscale 2 , 429-433 (2010).

60. Reed, B. W., Williams, D. R., Moser, B. P. \& Koski, K. J. Chemically tuning quantized acoustic phonons in $2 \mathrm{D}$ layered $\mathrm{MoO}_{3}$ nanoribbons. Nano Lett. 19, 4406-4412 (2019).

61. Liu, X. L., Zhang, X., Lin, M. L. \& Tan, P. H. Different angle-resolved polarization configurations of Raman spectroscopy: A case on the basal and edge plane of two-dimensional materials. Chinese Phys. B 26, 067802 (2017).

62. Py, M. A., Schmid, P. E. \& Vallin, J. T. Raman scattering and structural properties of $\mathrm{MoO}_{3}$. Nuovo Cim. B Ser. 11 38, 271-279 (1977).

63. $\mathrm{Kim}, \mathrm{J}$. H. et al. van der Waals epitaxial growth of single crystal $\mathrm{a}-\mathrm{MoO}_{3}$ layers on layered materials growth templates. 2D Mater. 6, 015016 (2018).

64. Puebla, S., Mariscal-jim, A., Munuera, C. \& Castellanos-gomez, A. Optical-based thickness measurement of $\mathrm{MoO}_{3}$ nanosheets. Nanomaterials 10, 1272 (2020).

65. Nikogosyan, D. N. Beta barium borate (BBO). Appl. Phys. A 52, 359-368 (1991).

66. G. Elert. Refraction - The Physics Hypertextbook. https://physics.info/refraction/ (1998).

67. Papadopoulos, N. et al. Large birefringence and linear dichroism in TiS 3 nanosheets. Nanoscale 10, 12424-12429 (2018). 
68. Iguiñiz, N., Frisenda, R., Bratschitsch, R. \& Castellanos-Gomez, A. Revisiting the buckling metrology method to determine the Young's modulus of $2 \mathrm{D}$ materials. Adv. Mater. 31, 1-24 (2019).

69. Feicht, P. et al. Systematic evaluation of different types of graphene oxide in respect to variations in their in-plane modulus. Carbon N.Y. 114, 700-705 (2017).

70. Kunz, D. A. et al. In-plane modulus of singular 2:1 clay lamellae applying a simple wrinkling technique. ACS Appl. Mater. Interfaces 5, 5851-5855 (2013).

71. Kunz, D. A. et al. Space-resolved in-plane moduli of graphene oxide and chemically derived graphene applying a simple wrinkling procedure. Adv. Mater. 25, 1337-1341 (2013).

72. Brennan, C. J., Nguyen, J., Yu, E. T. \& Lu, N. Interface adhesion between 2D materials and elastomers measured by Buckle Delaminations. Adv. Mater. Interfaces 2, 1-7 (2015).

73. Bowden, N., Brittain, S., Evans, A. G., Hutchinson, J. W. \& Whitesides, G. M. Spontaneous formation of ordered structures in thin films of metals supported on an elastomeric polymer. Nature 393, 146-149 (1998).

74. Terentjev, E. M. Precise determination of the Poisson ratio in soft materials with 2D digital image correlation. Soft Matter 9, 6037-6045 (2013).

75. Šiškins, M. et al. Highly anisotropic mechanical and optical properties of 2D layered As2S3 membranes. ACS Nano 13, 10845-10851 (2019).

76. Castellanos-Gomez, A. et al. Deterministic transfer of two-dimensional materials by all-dry viscoelastic stamping. 2D Mater. 1, 1-34 (2014).

77. Zhao, Q., Wang, T., Ryu, Y. K. \& Frisenda, R. An inexpensive system for the deterministic transfer of 2D materials. J. Phys. Mater. 3, 016001 (2020).

78. Frisenda, R. et al. Micro-reflectance and transmittance spectroscopy: a versatile and powerful tool to characterize 2D materials. J. Phys. D. Appl. Phys. 50, aa5256 (2017).

79. Giannozzi, P. et al. \{QUANTUM\} \{ESPRESSO\}: a modular and open-source software project for quantum simulations of materials. J. Phys. Condens. Matter 21, 395502 (2009).

80. Marini, A., Hogan, C., Gruening, M. \& Varsano, D. yambo: An ab initio tool for excited state calculations. Comput. Phys. Commun. 180, 1392-1403 (2009).

81. Sangalli, D. et al. Many-body perturbation theory calculations using the yambo code. J. Phys. Condens. Matter 31, 325902 (2019)

82. Perdew, J. P., Burke, K. \& Ernzerhof, M. Generalized gradient approximation made simple. Phys. Rev. Lett. 77, 3865-3868 (1996).

83. Hamann, D. R. Optimized norm-conserving Vanderbilt pseudopotentials. Phys. Rev. B 88, 85117 (2013)

84. Corso, A. D. https://dalcorso.github.io/thermo_pw/ (2020).

\section{ACKNOWLEDGEMENTS}

This project has received funding from the European Research Council (ERC) under the European Union's Horizon 2020 research and innovation programme (grant agreement $n^{\circ}$ 755655, ERC-StG 2017 project 2D-TOPSENSE), the European Commission, under the Graphene Flagship (Core 3, grant number 881603), the Spanish Ministry of Economy, Industry and Competitiveness through the grant MAT201787134-C2-2-R. R.F. acknowledges the support from the Spanish Ministry of Economy, Industry and Competitiveness (MINECO) through a Juan de la Cierva-formación fellowship 2017 FJCl-2017-32919. S.P. acknowledges the fellowship PRE2018-084818. R. D'A. acknowledges financial support from the grant Grupos Consolidados UPV/EHU del Gobierno Vasco (Grant No. IT1249-19), the support of the MICINN through the grant "SelectDFT" (Grant No. FIS2016-79464-P) and travel support from the MINECO grant "TowTherm" (Grant No. MINECOG17/A01). G.S.-S. acknowledges financial support from Spanish MICIU RTI2018-099054-J-I00 and MICINN IJC2018-038164-I. Electron microscopy observations were carried out at the Centro Nacional de Microscopia Electronica, CNME-UCM.

\section{AUTHOR CONTRIBUTIONS}

S.P. fabricated the $\mathrm{MoO}_{3}$ flake samples and performed the optical microscopy, Raman spectroscopy and buckling metrology experiments. G.S-S., performed the electron microscopy experiments. R.F., C.M. and A.C.-G. supervised and designed the experiments. S.P., C.M. and A.C.-G. drafted the firsts version of the manuscript. R.D'A. developed the theoretical calculations. All authors contributed to writing the final version of the manuscript.

\section{COMPETING INTERESTS}

The authors declare no competing interests.

\section{ADDITIONAL INFORMATION}

Supplementary information The online version contains supplementary material available at https://doi.org/10.1038/s41699-021-00220-5.

Correspondence and requests for materials should be addressed to A.C-G.

Reprints and permission information is available at http://www.nature.com/ reprints

Publisher's note Springer Nature remains neutral with regard to jurisdictional claims in published maps and institutional affiliations.

\begin{abstract}
Open Access This article is licensed under a Creative Commons Attribution 4.0 International License, which permits use, sharing adaptation, distribution and reproduction in any medium or format, as long as you give appropriate credit to the original author(s) and the source, provide a link to the Creative Commons license, and indicate if changes were made. The images or other third party material in this article are included in the article's Creative Commons license, unless indicated otherwise in a credit line to the material. If material is not included in the article's Creative Commons license and your intended use is not permitted by statutory regulation or exceeds the permitted use, you will need to obtain permission directly from the copyright holder. To view a copy of this license, visit http://creativecommons. org/licenses/by/4.0/.
\end{abstract}

(c) The Author(s) 2021 